\title{
Spur gear generation by shaper cutters
}

\author{
Chung-Biau Tsay ${ }^{*}$, Wen-Yao Liu, Yi-Cheng Chen \\ Department of Mechanical Engineering, National Chiao Tung University, Hsinchu 300, Taiwan, ROC
}

Received 2 March 1999

\begin{abstract}
This paper proposes the computerized tooth profile generation of spur gears cut with shaper cutters. A complete geometrical mathematical model of a spur shaper cutter is proposed, including the protuberance, the involute region and semi-topping. The profiles of standard or non-standard spur gears cut with the shaper cutter are obtained based on the theory of gearing. In addition, the effects of the cutter parameters on the generated tooth profile are investigated and illustrated with computer simulation, while the appropriate shaper cutter parameters corresponding to the required gear profile, are also determined using an optimization method. (C) 2000 Elsevier Science S.A. All rights reserved.
\end{abstract}

Keywords: Spur gear; Shaper cutter; Mathematical model; Protuberance; Undercut; Optimization

\section{Introduction}

Gear shaping is a versatile and accurate means of manufacturing spur gears, helical gears, herringbone gears, internal gears and face gears. Although resembling a spur pinion or gear in appearance, a spur shaper cutter has eliminated cutting edges and increased addenda. In addition, the teeth of a spur shaper cutter have zero degrees of helix angle, and the teeth are aligned parallel to the cutter axis. Many gear handbooks describe the shaper cutter in detail.

Tooth profile modifications for specific purposes are frequently applied on gear cutters such as racks, hobs and shapers, among which, semi-topping and protuberance are two common modifications. A semi-topping form is used to produce a tip chamfer on the gear. Also, a ramp is added to the cutter root to produce a reasonably uniform chamfer at the tip of the gear tooth. On the other hand, having a small undercut in the prefinished gear is desired when finishing a gear by shaving or grinding. Notably, an intentional undercut can be made near the gear root by using a protuberance-type cutter.

Several investigations have made tooth modifications on rack cutters, including protuberance and semi-topping. Vijayakar et al. [1] developed gear tooth profiles from an arbitrary geometry of a rack cutter with protuberance and root chamfer. Later, Ariga and Nagata [2] studied

\footnotetext{
*Corresponding author. Tel./fax: +886-3-572-840. E-mail address: cbtsay@cc.nctu.edu.tw (C.-B. Tsay).
}

the chamfer produced by semi-topping on hob and rack cutters. More recently, Kuang and Chen [3] determined the protuberance dimensions on a rack cutter to provide an adequate undercut for a standard or non-standard spur gear.

Tooth profiles cut with pinion cutters have also received considerable interest. Green and Mabie [4] presented a method of designing non-standard spur gears cut with a pinion cutter, making the static bending strengths in the pinion and in the gear approximately equal. Rogers et al. [5] investigated the values of pinion cutter offsets for nonstandard spur gears to satisfy two design constraints: maximum recess to the approach action or equalizing the tooth strengths for a spur gear pair. In addition, Yoshino et al. [6] calculated the grinding wheel profiles for finishing pinion cutters for concave Novikov gears. Kim and Kim [7] developed a process and a computer software to design a pinion cutter. Chang and Tsay [8] analyzed the tooth profile and undercutting conditions of non-circular gears generated by shaper cutters. However, their investigations consider the tooth modifications on shaper cutters. Janninck $[9,10]$ introduced the basic concepts for the design and application of shaper cutters, while considering protuberance and semitopping. However, that investigation did not quantitatively determine the relationship between the cutter parameters and the generated tooth profiles.

Most gear-shaping-related investigations have largely focused on the working parts of the gear teeth. In this study, the authors investigate the profiles of the shaper cutter and the generated gear. A complete profile of the shaper cutter is 
initially constructed, including semi-topping, protuberance and involute curve. Based on the gear generation mechanism and the theory of gearing [11], a mathematical model of the generated gear is then derived, including the tip chamfer, working part, fillet and undercut. Moreover, the relationship between the shaper cutter parameters and the tooth profile is elucidated with illustrative graphs. Finally, an optimization method is applied to estimate the proper parameters of a shaper cutter to achieve the gear's prescribed feature.

\section{Geometry of shaper cutters}

A spur shaper cutter resembles a spur pinion or gear in appearance. For simplicity, the generation of spur gears with shaper cutters can be simplified into a two-dimensional problem. As shown in Fig. 1, the profile of a shaper cutter contains four major regions. Region 1 of the shaper cutter is an involute curve AD which generates the working part of the spur gears. Region 2 is a straight line DE near the tooth root which forms the so-called semi-topping. Semi-topping attempts to generate a tip chamfer on the generated tooth surface to avoid interference during the meshing process. Region 3 is a straight line $\mathrm{AB}$ and region 4 is a circular curve BC. Regions 3 and 4 cumulatively form the protuberance to produce an adequate amount of undercut on the generated fillet. The intentional undercut is prepared for further precise finishing after shaping, such as shaving, skiving or grinding. In Fig. 1 , coordinates systems $S_{\mathrm{s}}\left(X_{\mathrm{s}}, Y_{\mathrm{s}}\right)$ and $S_{\mathrm{c}}\left(X_{\mathrm{c}}, Y_{\mathrm{c}}\right)$ represent the reference and shaper cutter coordinate systems, respectively. The equations of regions $1-4$, represented in coordinate systems $S_{\mathrm{s}}$ and $S_{\mathrm{c}}$, are presented as follows.

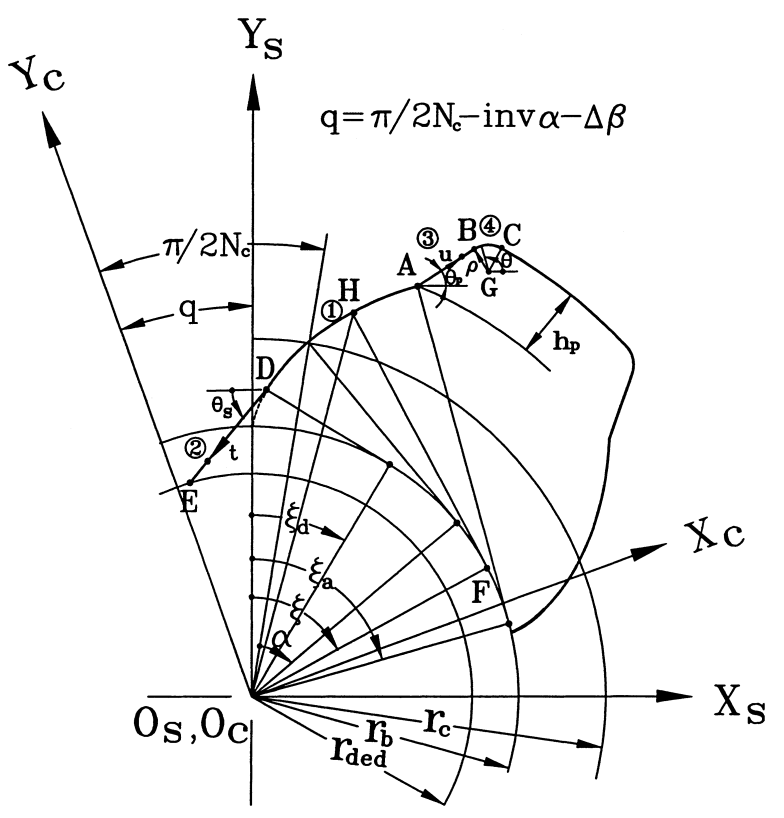

Fig. 1. Geometry of the shaper cutter.

\subsection{Region 1}

Region 1 is an involute curve used to generate the working tooth surface of the gear. As shown in Fig. 1, $r_{\mathrm{b}}$ denotes the radius of the base circle of the shaper cutter, $\xi$ represents a design parameter (i.e. the involute polar angle) of the shaper cutter which determines the position of point $\mathrm{H}$ on the involute curve $\mathrm{AD}$, and $r_{\mathrm{c}}$ and $r_{\mathrm{ded}}$ are the pitch radius and dedendum radius of the shaper cutter, respectively. The equation of region 1 , represented in coordinate system $S_{\mathrm{s}}$, can be expressed as

$\boldsymbol{R}_{\mathrm{s}}^{(1)}=\left\{\begin{array}{c}x_{\mathrm{s}}^{(1)} \\ y_{\mathrm{s}}^{(1)}\end{array}\right\}=\left\{\begin{array}{l}r_{\mathrm{b}} \sin \xi-r_{\mathrm{b}} \xi \cos \xi \\ r_{\mathrm{b}} \cos \xi+r_{\mathrm{b}} \xi \sin \xi\end{array}\right\}$.

According to the relationship between coordinate systems $S_{\mathrm{s}}$ and $S_{\mathrm{c}}$ illustrated in Fig. 1, the position vector of regions $1-4$ can be transformed from coordinate systems $S_{\mathrm{s}}$ to $S_{\mathrm{c}}$ by applying the following homogeneous coordinate transformation matrix equation:

$\boldsymbol{R}_{\mathrm{c}}^{(i)}=\left[M_{\mathrm{cs}}\right] \boldsymbol{R}_{\mathrm{s}}^{(i)}$,

where

$\left[M_{\mathrm{cs}}\right]=\left[\begin{array}{cc}\cos q & \sin q \\ -\sin q & \cos q\end{array}\right]$

$q=\frac{\pi}{2 N_{\mathrm{c}}}-\operatorname{inv} \alpha-\Delta \beta$.

In Eqs. (2) and (3), parameters $N_{\mathrm{c}}$ and $\alpha$ denote the teeth number and pressure angle of the shaper cutter, respectively; and superscripts $i=1-4$ indicate the regions $1-4$ on the shaper cutter, respectively. When the backlash angle $\Delta \beta$ is positive, the backlash indeed exists in the generated gear. Substituting Eq. (1) in Eq. (2) yields the position vector of region 1 represented in coordinate system $S_{\mathrm{c}}$ as follows:

$\boldsymbol{R}_{\mathrm{c}}^{(1)}=\left\{\begin{array}{l}r_{\mathrm{b}} \sin (\xi+q)-r_{\mathrm{b}} \xi \cos (\xi+q) \\ r_{\mathrm{b}} \cos (\xi+q)+r_{\mathrm{b}} \xi \sin (\xi+q)\end{array}\right\}$.

Based on the differential geometry, the unit normal vectors of regions 1-4 of the shaper cutter surface represented in coordinate system $S_{\mathrm{c}}$ can be obtained by

$\boldsymbol{n}_{\mathrm{c}}^{(i)}=\frac{\boldsymbol{N}_{\mathrm{c}}^{(i)}}{\left|\boldsymbol{N}_{\mathrm{c}}^{(i)}\right|}$,

where

$\boldsymbol{N}_{\mathrm{c}}^{(i)}=\frac{\partial \boldsymbol{R}_{\mathrm{c}}^{(i)}}{\partial \theta_{\mathrm{c}}^{(i)}} \times \boldsymbol{k}_{\mathrm{c}}$

$\boldsymbol{k}_{\mathrm{c}}$ is the unit vector of the $Z_{\mathrm{c}}$-axis, $\theta_{\mathrm{c}}$ represents the tooth profile parameter of the shaper cutter, and superscripts $i=1-4$ indicate regions $1-4$ on the shaper cutter, respectively. Substituting Eq. (4) in Eq. (5) yields the unit normal vector of region 1 represented in coordinate system $S_{\mathrm{c}}$ as follows:

$\boldsymbol{n}_{\mathrm{c}}^{(1)}=\left\{\begin{array}{c}\cos (\xi+q) \\ -\sin (\xi+q)\end{array}\right\}$. 


\subsection{Region 2}

Region 2 of the shaper cutter is the semi-topping and is represented by the straight line DE. According to Fig. 1, symbol $\xi_{\mathrm{d}}$ is the involute polar angle of point $\mathrm{D}$, the start point of the semi-topping region. The coordinates of point $\mathrm{D}$ represented in coordinate system $S_{\mathrm{c}}$ are

$\left\{\begin{array}{c}D_{\mathrm{s} x} \\ D_{\mathrm{s} y}\end{array}\right\}=\left\{\begin{array}{l}r_{\mathrm{b}} \sin \xi_{\mathrm{d}}-r_{\mathrm{b}} \xi_{\mathrm{d}} \cos \xi_{\mathrm{d}} \\ r_{\mathrm{b}} \cos \xi_{\mathrm{d}}+r_{\mathrm{b}} \xi_{\mathrm{d}} \sin \xi_{\mathrm{d}}\end{array}\right\}$.

Therefore, the position vector of region 2 represented in coordinate system $S_{\mathrm{c}}$ can be obtained as follows:

$\boldsymbol{R}_{\mathrm{s}}^{(2)}=\left\{\begin{array}{c}x_{\mathrm{s}}^{(2)} \\ y_{\mathrm{s}}^{(2)}\end{array}\right\}=\left\{\begin{array}{c}D_{\mathrm{s} x}+t \cos \theta_{\mathrm{s}} \\ D_{\mathrm{s} y}+t \sin \theta_{\mathrm{s}}\end{array}\right\}$,

where $\theta_{\mathrm{s}}$ denotes the angle formed by a horizontal line and line $\mathrm{DE}, t$ represents the position on the semi-topping region, and is limited by $t_{\max } \geq t \geq 0$.

Similarly, region 2 of the shaper cutter can be transformed into coordinate system $S_{\mathrm{c}}$ and represented as follows:

$\boldsymbol{R}_{\mathrm{c}}^{(2)}=\left\{\begin{array}{l}D_{\mathrm{s} x} \cos q+D_{\mathrm{s} y} \sin q+t \cos \left(\theta_{\mathrm{s}}-q\right) \\ -D_{\mathrm{s} x} \sin q+D_{\mathrm{s} y} \cos q+t \sin \left(\theta_{\mathrm{s}}-q\right)\end{array}\right\}$.

By substituting Eq. (9) in Eq. (5), the unit normal vector of region 2 can be obtained as follows:

$\boldsymbol{n}_{\mathrm{c}}^{(2)}=\left\{\begin{array}{c}\sin \left(\theta_{\mathrm{s}}-q\right) \\ -\cos \left(\theta_{\mathrm{s}}-q\right)\end{array}\right\}$.

\subsection{Region 3}

Region 3 is the straight line $\mathrm{AB}$ of the protuberance on the shaper cutter, as shown in Fig. 1. The position vector of region 3 represented in coordinate system $S_{\mathrm{s}}$ is

$\boldsymbol{R}_{\mathrm{s}}^{(3)}=\left\{\begin{array}{l}x_{\mathrm{s}}^{(3)} \\ y_{\mathrm{s}}^{(3)}\end{array}\right\}=\left\{\begin{array}{l}A_{\mathrm{s} x}+u \cos \theta_{\mathrm{p}} \\ A_{\mathrm{s} y}+u \sin \theta_{\mathrm{p}}\end{array}\right\}$,

where $A_{\mathrm{s} x}$ and $A_{\mathrm{s} y}$ are the coordinates of point A represented in coordinate system $S_{\mathrm{s}}, \theta_{\mathrm{p}}$ represents the angle formed by the straight line $\mathrm{AB}$ and a horizontal line, and $u$ is a design parameter indicating the position on the straight line $\mathrm{AB}$ and is limited by $0 \leq u \leq u_{\max }$. The position vector and the unit normal vector of region 3 represented in coordinate system $S_{\mathrm{c}}$ can be expressed as follows:

$\boldsymbol{R}_{\mathrm{s}}^{(3)}=\left\{\begin{array}{l}A_{\mathrm{s} x} \cos q+A_{\mathrm{s} y} \sin q+u \cos \left(\theta_{\mathrm{p}}-q\right) \\ -A_{\mathrm{s} x} \sin q+A_{\mathrm{s} y} \cos q+u \sin \left(\theta_{\mathrm{p}}-q\right)\end{array}\right\}$,

$\boldsymbol{n}_{\mathrm{c}}^{(3)}=\left\{\begin{array}{c}\sin \left(\theta_{\mathrm{p}}-q\right) \\ -\cos \left(\theta_{\mathrm{p}}-q\right)\end{array}\right\}$.

\subsection{Region 4}

Region 4, represented by a circular curve BC in Fig. 1, is the rounded tip of the protuberance. The circular curve $\mathrm{BC}$ is centered at point $\mathrm{G}$ with radius $\rho$. Thus, the position vector of region 4 represented in coordinate system $S_{\mathrm{s}}$ is as follows:

$\boldsymbol{R}_{\mathrm{s}}^{(4)}=\left\{\begin{array}{l}x_{\mathrm{s}}^{(4)} \\ y_{\mathrm{s}}^{(4)}\end{array}\right\}=\left\{\begin{array}{l}G_{\mathrm{s} x}+\rho \cos \theta \\ G_{\mathrm{s} y}+\rho \sin \theta\end{array}\right\}$,

where $G_{\mathrm{s} x}$ and $G_{\mathrm{s} y}$ are the coordinates of point $\mathrm{G}$ represented in coordinate system $S_{\mathrm{s}}, \theta$ is a design parameter indicating the position on circular curve $\mathrm{BC}$ and is limited by $\theta_{\mathrm{c}} \leq \theta \leq \theta_{\mathrm{B}}, \theta_{\mathrm{B}}$ is the angle formed by line GB and a horizontal line, and $\theta_{\mathrm{c}}$ is the angle formed by line GC and a horizontal line. Consequently, the position vector and unit normal vector of region 4 of the shaper cutter can be expressed in coordinate system $S_{\mathrm{c}}$ as follows:

$\boldsymbol{R}_{\mathrm{c}}^{(4)}=\left\{\begin{array}{l}G_{\mathrm{s} x} \cos q+G_{\mathrm{s} y} \sin q+\rho \cos (\theta-q) \\ -G_{\mathrm{s} x} \sin q+G_{\mathrm{s} y} \cos q+\rho \sin (\theta-q)\end{array}\right\}$,

$\boldsymbol{n}_{\mathrm{c}}^{(4)}=\left\{\begin{array}{l}\sin \theta \\ \cos \theta\end{array}\right\}$.

\section{Mathematical model of the generated spur gears}

Gear shaping is a generation method. Fig. 2 depicts the kinematic relationship between the shaper cutter and the gear generated during the manufacturing process. Coordinate systems $S_{\mathrm{c}}\left(X_{\mathrm{c}}, Y_{\mathrm{c}}, Y_{\mathrm{c}}\right)$ and $S_{\mathrm{g}}\left(X_{\mathrm{g}}, Y_{\mathrm{g}}, Y_{\mathrm{g}}\right)$ are rigidly attached to the shaper cutter and the gear, respectively, point I is the instantaneous center of rotation, $\phi_{\mathrm{c}}$ and $\phi_{\mathrm{g}}$ are the rotation angles of the shaper cutter and the gear, respectively, and $r_{\mathrm{c}}$ and $r_{\mathrm{g}}$ are the standard pitch radii of the shaper cutter and the gear, respectively. When the profile-shifted amount is zero, i.e. $\delta x=0$, the center distance between the shaper cutter and the gear is $C=r_{\mathrm{c}}+r_{\mathrm{g}}$. The position vector of the shaper cutter can be transformed from coordinate systems $S_{\mathrm{c}}$ to $S_{\mathrm{g}}$ by applying the following homogeneous coordinate transformation matrix equation:

$\boldsymbol{R}_{\mathrm{g}}^{(i)}=\left[M_{\mathrm{gc}}\right] \boldsymbol{R}_{\mathrm{c}}^{(i)}$,

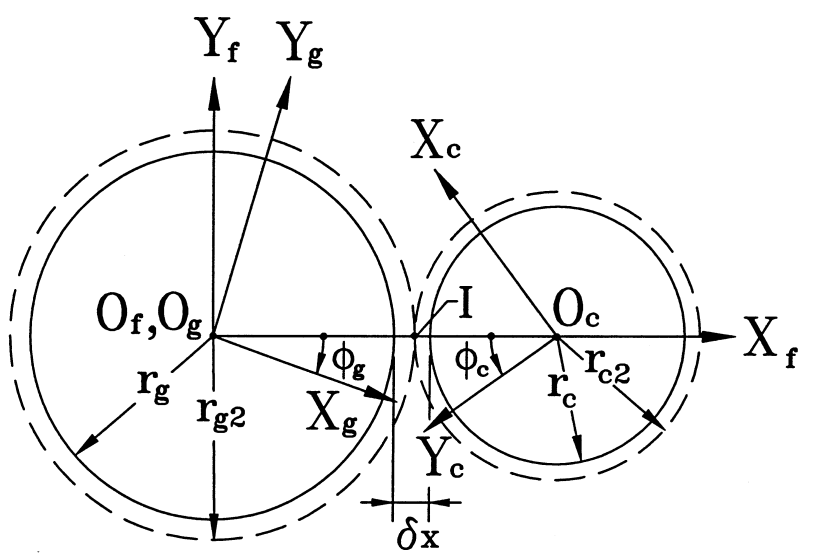

Fig. 2. Kinematic relationship between the shaper cutter and the generated gear. 
$\left[M_{\mathrm{gc}}\right]=\left[M_{\mathrm{gf}}\right]\left[M_{\mathrm{gf}}\right]=\left[\begin{array}{ccc}-\sin \phi & -\cos \phi & C \cos \phi_{\mathrm{g}} \\ \cos \phi & -\sin \phi & C \sin \phi_{\mathrm{g}} \\ 0 & 0 & 1\end{array}\right]$,

where $\left[M_{\mathrm{gf}}\right]$ and $\left[M_{\mathrm{fc}}\right]$ are the homogeneous transformation matrices transforming from coordinate systems $S_{\mathrm{f}}$ to $S_{\mathrm{g}}$ and from $S_{\mathrm{c}}$ to $S_{\mathrm{f}}$, respectively, and $\phi=\phi_{\mathrm{c}}+\phi_{\mathrm{g}}$.

The equation of meshing of the shaper and the generated gear can be represented as follows [11]:

$\frac{X_{\mathrm{c}}^{(i)}-x_{\mathrm{c}}^{(i)}}{n_{\mathrm{xc}}^{(i)}}=\frac{Y_{\mathrm{c}}^{(i)}-y_{\mathrm{c}}^{(i)}}{n_{\mathrm{yc}}^{(i)}}$.

In Eqs. (17) and (18), superscript $i$ indicates regions 1-4 on the shaper cutter, $X_{\mathrm{c}}^{(i)}$ and $Y_{\mathrm{c}}^{(i)}$ denote the coordinates of the instantaneous center of rotation I, represented in coordinate system $S_{\mathrm{c}}$, and $n_{x \mathrm{c}}^{(i)}$ and $n_{y \mathrm{c}}^{(i)}$ are the directional components of the unit normal $\boldsymbol{n}_{\mathrm{c}}^{(i)}$ along the $X_{\mathrm{c}}$ and $Y_{\mathrm{c}}$ axes, respectively.

Based on the theory of gearing, the profile of the gear generated can be obtained by simultaneously considering the loci of the shaper cutter represented in the gear's coordinate system $S_{\mathrm{g}}$ and the equation of meshing.

1. Working part of the gear.

The working part of the generated gear is an involute curve generated by region 1 (involute curve) of the shaper cutter. By substituting Eqs. (4) and (6) in Eqs. (17) and (18), the mathematical model of the working part on the spur gear is obtained as follows:

$\boldsymbol{R}_{\mathrm{g}}^{(1)}=\left\{\begin{array}{l}-r_{\mathrm{b}} \cos (\xi+q-\phi)-r_{\mathrm{b}} \xi \sin (\xi+q-\phi)+C \cos \phi_{\mathrm{g}} \\ r_{\mathrm{b}} \sin (\xi+q-\phi)-r_{\mathrm{b}} \xi \cos (\xi+q-\phi)+C \sin \phi_{\mathrm{g}}\end{array}\right\}$,

$\phi_{\mathrm{c}}=-\alpha+\xi+q$.

The second equation in Eq. (19) is the so-called equation of meshing.

2. Chamfer of the gear.

The tip chamfer on the generated spur gear is generated by region 2 (semi-topping) on the shaper cutter. Substituting Eqs. (9) and (10) in Eqs. (17) and (18) yields the equation of the chamfer on the spur gear as follows:

$$
\begin{aligned}
& \boldsymbol{R}_{\mathrm{g}}^{(2)}=\left\{\begin{array}{l}
D_{\mathrm{s} x} \sin (q-\phi)-D_{\mathrm{s} y} \cos (q-\phi)-t \sin \left(\theta_{\mathrm{s}}+\phi-q\right)+C \cos \phi_{\mathrm{g}} \\
D_{\mathrm{s} x} \cos (q-\phi)+D_{\mathrm{s} y} \sin (q-\phi)+t \cos \left(\theta_{\mathrm{s}}+\phi-q\right)+C \sin \phi_{\mathrm{g}}
\end{array}\right\}, \\
& r_{\mathrm{c}} \sin \left(\phi_{\mathrm{c}}+\theta_{\mathrm{s}}-q\right)=D_{\mathrm{s} x} \cos \theta_{\mathrm{s}}+D_{\mathrm{s} y} \sin \theta_{\mathrm{s}}+t .
\end{aligned}
$$

3. Surface generated by region 3 (straight line) of the shaper cutter.

As mentioned earlier, region 3 on the shaper cutter is the straight line of the protuberance. Similarly, by substituting Eqs. (12) and (13) in Eqs. (17) and (18), the tooth surface generated by region 3 of the shaper cutter can be expressed by

$$
\begin{aligned}
& \boldsymbol{R}_{\mathrm{g}}^{(3)}=\left\{\begin{array}{l}
A_{\mathrm{s} x} \sin (q-\phi)-A_{\mathrm{sy}} \cos (q-\phi)-u \sin \left(\theta_{\mathrm{s}}+\phi-q\right)+C \cos \phi_{\mathrm{g}} \\
A_{\mathrm{s} x} \cos (q-\phi)+A_{\mathrm{s} y} \sin (q-\phi)+u \cos \left(\theta_{\mathrm{s}}+\phi-q\right)+C \sin \phi_{\mathrm{g}}
\end{array}\right\}, \\
& r_{\mathrm{c}} \sin \left(\phi_{\mathrm{c}}+\theta_{\mathrm{p}}-q\right)=A_{\mathrm{s} x} \cos \theta_{\mathrm{p}}+A_{\mathrm{s} y} \sin \theta_{\mathrm{p}}+u .
\end{aligned}
$$

4. Surface generated by region 4 (circular-curve) of the shaper cutter.

Similarly, the tooth surface generated by region 4 on the shaper cutter can be obtained by substituting Eqs. (15) and (16) in Eqs. (17) and (18), and expressed as follows:

$$
\begin{aligned}
& \boldsymbol{R}_{\mathrm{g}}^{(4)}=\left\{\begin{array}{l}
G_{\mathrm{s} x} \sin (q-\phi)-G_{\mathrm{sy}} \cos (q-\phi)-\rho \sin (\phi+\theta-q)+C \cos \phi_{\mathrm{g}} \\
G_{\mathrm{s} x} \cos (q-\phi)+G_{\mathrm{s} y} \sin (q-\phi)+\rho \cos (\phi+\theta-q)+C \sin \phi_{\mathrm{g}}
\end{array}\right\}, \\
& r_{\mathrm{c}} \cos \left(\phi_{c}+\theta\right)=-G_{\mathrm{s} x} \sin \theta+G_{\mathrm{s} y} \cos \theta .
\end{aligned}
$$

\section{Non-standard teeth profiles}

Profile-shifted gears are applied to the gear generation under several conditions such as non-standard center distance or an attempt to avoid tooth undercutting. Fig. 2 also depicts the relationship between the shaper cutter and the generated gear when the shaper cutter is shifted by an amount $\delta x=x m$, where $x$ denotes the profile shifting coefficient and $m$ represents the module of the shaper/gear. As mentioned earlier, $r_{\mathrm{g}}$ and $r_{\mathrm{c}}$ are the standard pitch radii of the gear and the shaper cutter, respectively. The new center distance becomes $C_{2}=r_{\mathrm{c}}+r_{\mathrm{g}}+\delta x$ when the amount of shift $\delta x$ is present. Since the teeth numbers of the shaper and the gear, $N_{\mathrm{c}}$ and $N_{\mathrm{g}}$, remain unchanged, the new pitch radii of the shaper cutter and the gear when the amount of shift $\delta x$ is present become

$r_{\mathrm{c} 2}=C_{2} \frac{N_{\mathrm{c}}}{N_{\mathrm{g}}}$,
$r_{\mathrm{g} 2}=C_{2} \frac{N_{\mathrm{g}}}{N_{\mathrm{c}}}$.

Hence, the mathematical model of a profile-shifted gear can be attained by substituting the new pitch radii of the shaper cutter $r_{\mathrm{c} 2}$ and gear $r_{\mathrm{g} 2}$ into the previously developed mathematical models.

\section{Investigation on shaper cutter parameters}

In Sections 2 and 3, the complete mathematical models of the shaper cutter and the generated spur gear have been derived. Based on the mathematical models derived therein, computer simulation programs have also been developed for the tooth profile generation. In practice, the proper shaper cutter parameters are usually estimated according to the desired gear profiles. In this section, several numerical examples are studied to investigate how the shaper cutter parameters affect the generated tooth profile. In addition, the optimization method is adopted to determine adequate shaper cutter parameters to meet the prescribed gear profile.

\subsection{Tooth profile parameters}

Several parameters of the generated tooth profile must be initially defined to quantitatively describe the generated 


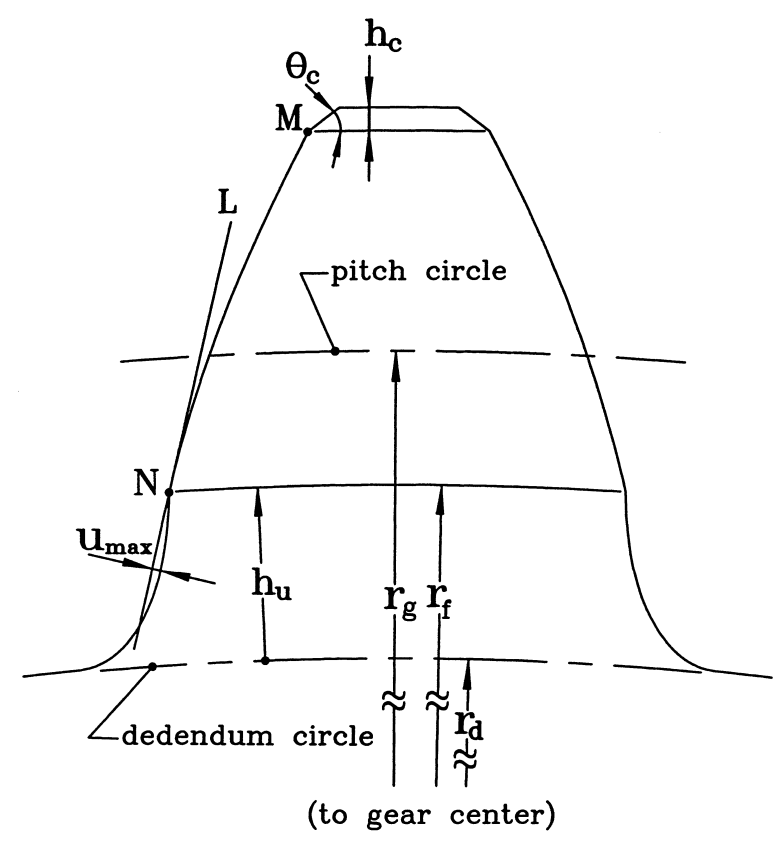

Fig. 3. Parameters of the generated tooth profile.

tooth profile. Fig. 3 depicts a generated tooth profile and its profile parameters. In Fig. 3, curve MN is the working part on the generated gear. The coordinates of point $\mathrm{M}$ (initial point of chamfer) can be determined by solving the nonlinear equations of the chamfer region and the involute region simultaneously. Similarly, the coordinates of point $\mathrm{N}$ (the initial point of the fillet) can be determined by solving the non-linear equations of the involute region and the fillet region simultaneously. In the chamfer region, $h_{\mathrm{c}}$ denotes the height of the chamfer and $\theta_{\mathrm{c}}$ represents the chamfer angle, formed by a horizontal line and the chamfer. In the fillet region, line $\mathrm{L}$ is the tangent to point $\mathrm{N}, r_{\mathrm{f}}$ denotes the radius of point $\mathrm{N}, r_{\mathrm{d}}$ represents the dedendum radius of the gear, and $h_{\mathrm{u}}=r_{\mathrm{f}}-r_{\mathrm{d}}$. The amount of undercut can be defined by the perpendicular distance measured from the undercut curve to the line $\mathrm{L}$, and $u_{\max }$ is defined as the maximum amount of undercut. Obviously, a larger $h_{\mathrm{u}}$ leads to a shorter working part and therefore a smaller contact ratio of the gear.

\subsection{Effects of shaper cutter parameters on the generated tooth profile}

The tip chamfer and the fillet of the gear tooth are generated by the semi-topping and the protuberance of the shaper cutter, respectively. Therefore, investigations on the effects of shaper cutter parameters on the tooth profile fall into two parts: the effect on the chamfer region and the effect on the fillet region. Table 1 lists some major design parameters of the shaper cutter and the generated gear employed in the following investigations.
Table 1

Some major design parameters for the shaper cutter and the generated gear

\begin{tabular}{ll}
\hline Basic parameters & \\
Module, $m$ & $10 \mathrm{~mm}$ \\
Teeth number & 25 \\
Pressure angle & $20^{\circ}$ \\
Backlash angle, $\Delta \beta$ & $0^{\circ}$ \\
Protuberance parameters & \\
$\theta_{\mathrm{p}}$ & $5^{\circ}$ \\
$h_{\mathrm{p}}$ & $0.15 \mathrm{~m}$ \\
$\rho$ & $0.3 h_{\mathrm{p}}$ \\
Semi-topping parameters & \\
$\xi_{\mathrm{d}}$ & $0^{\circ}$ \\
$\theta_{\mathrm{s}}$ & $45^{\circ}$ \\
Shift coefficient & \\
$\delta x$ & 0 \\
Gear parameter & \\
Teeth number & 30 \\
\hline
\end{tabular}

\subsection{Example 1: effects of semi-topping parameters on the generated tip chamfer}

According to Fig. 3, the tip chamfer on the generated tooth profile has two parameters, $\theta_{\mathrm{c}}$ and $h_{\mathrm{c}}$, denoting the angle and height of the chamfer, respectively. On the other hand, as shown in Fig. 1, the semi-topping on the shaper cutter also has two parameters $\theta_{\mathrm{s}}$ and $\xi_{\mathrm{d}}$. Parameter $\theta_{\mathrm{s}}$ is the angle formed between the semi-topping and a horizontal line and parameter $\xi_{\mathrm{d}}$ is the involute polar angle of point D. Based on the developed mathematical model of the generated gear tooth and the design parameters listed in Table 1, the effects of parameters $\xi_{\mathrm{d}}$ and $\theta_{\mathrm{s}}$ on the generated tooth profile are investigated and illustrated in Fig. 4. Meanwhile, Fig. 5 summarizes the effects of $\xi_{\mathrm{d}}$ and $\theta_{\mathrm{s}}$ on the two chamfer parameters, $\theta_{\mathrm{c}}$ and $h_{\mathrm{c}}$. According to Fig. 5(a), although $\xi_{\mathrm{d}}$ increases with an increase of $h_{\mathrm{c}}, \theta_{\mathrm{c}}$ remains nearly unchanged. As Fig. 5(b) indicates, $\theta_{\mathrm{c}}$ increases but $h_{\mathrm{c}}$ decreases as $\theta_{\mathrm{s}}$ increases. In sum, the parameter $\xi_{\mathrm{s}}$ affects the chamfer height $h_{\mathrm{c}}$ while the semi-topping angle $\theta_{\mathrm{s}}$ dominates the chamfer angle $\theta_{\mathrm{c}}$.

\subsection{Example 2: effects of protuberance parameters on the generated fillet and undercut}

According to Fig. 3, two parameters, $u_{\max }$ and $h_{\mathrm{u}}$ are relevant to the undercut and fillet region of the generated tooth profile, which are affected by the three protuberance parameters $\rho, h_{\mathrm{p}}$ and $\theta_{\mathrm{p}}$ of the shaper cutter, as shown in Fig. 1. Parameter $\rho$ refers to the radius of curve $\mathrm{BC}, h_{\mathrm{p}}$ denotes the height of the protuberance, and $\theta_{\mathrm{p}}$ represents the angle formed by line $\mathrm{AB}$ and a horizontal line. Several numerical examples are applied to investigate how the three shaper cutter parameters $\rho, h_{\mathrm{p}}$ and $\theta_{\mathrm{p}}$ affect the tooth profile parameters $u_{\max }$ and $h_{\mathrm{u}}$, individually. First, the effect of 
(a)

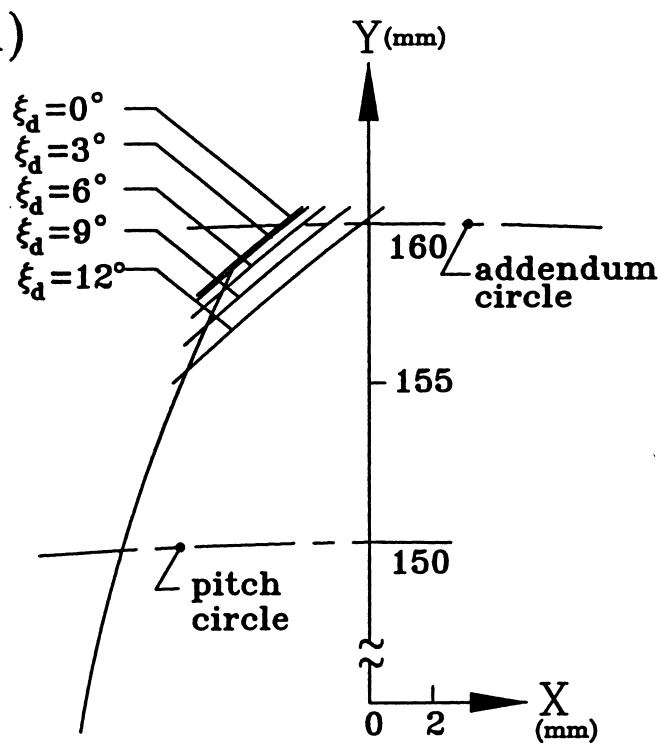

(b)

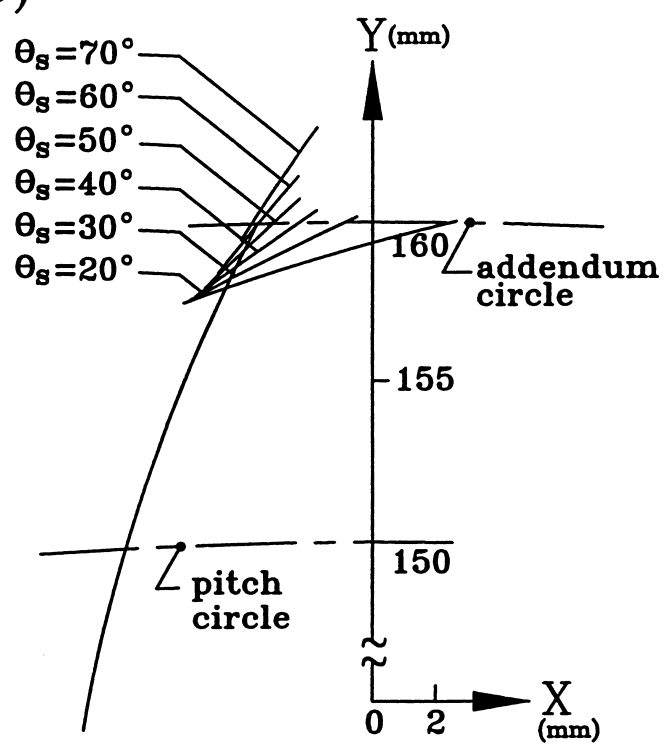

Fig. 4. Effect of semi-topping parameters $\xi_{\mathrm{d}}$ and $\theta_{\mathrm{s}}$ on the generated tooth profile.

different $\rho$ on the generated tooth profile and on the two parameters $u_{\max }$ and $h_{\mathrm{u}}$ are investigated and illustrated in Figs. 6 and 7, respectively. Fig. 7 reveals that increasing the radius $\rho$ decreases $u_{\max }$ and $h_{\mathrm{u}}$. This finding suggests that a larger radius $\rho$ of the shaper cutter protuberance results in a smaller undercut. In addition, Figs. 8 and 9 illustrate the effect of protuberance parameter $h_{\mathrm{p}}$ on the generated tooth profile and on the two parameters $u_{\max }$ and $h_{\mathrm{u}}$, respectively. According to Fig. 9, both parameters $u_{\max }$ and $h_{\mathrm{u}}$ increase with an increase of $h_{\mathrm{p}}$. Obviously, the amount of undercut increases with an increase of the protuberance height $h_{\mathrm{p}}$. Finally, the effect of protuberance parameter $\theta_{\mathrm{p}}$ on the generated tooth profile and on the two parameters $u_{\max }$ and $h_{\mathrm{u}}$ are illustrated in Figs. 10 and 11, respectively. (a) $\theta$

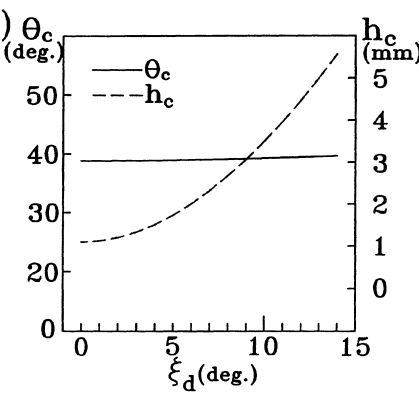

(b)

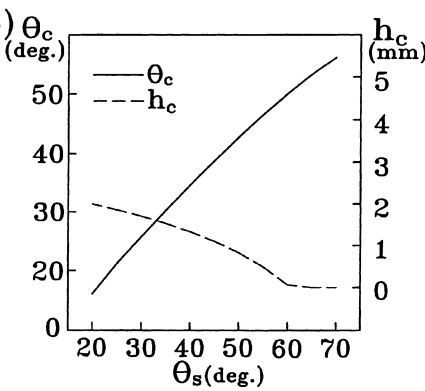

Fig. 5. Effect of semi-topping parameters $\xi_{\mathrm{d}}$ and $\theta_{\mathrm{s}}$ on the chamfer parameters $\theta_{\mathrm{c}}$ and $h_{\mathrm{c}}$.

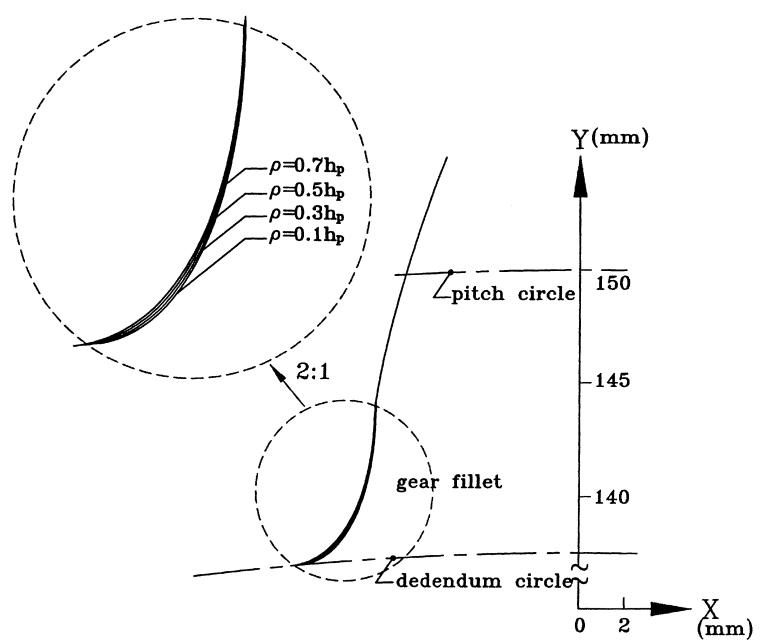

Fig. 6. Effect of protuberance parameter $\rho$ on the generated tooth profile.

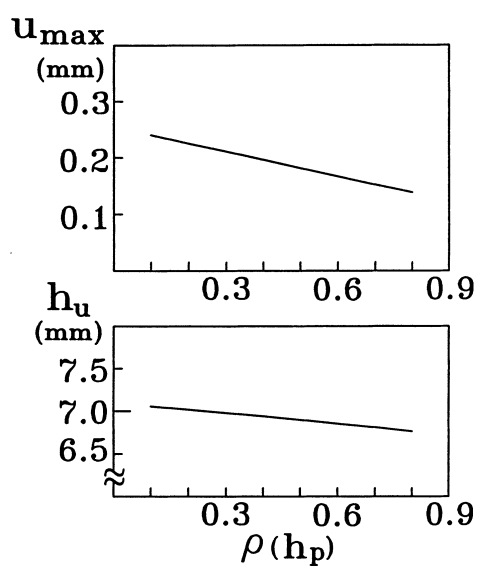

Fig. 7. Effects of protuberance parameter $\rho$ on the fillet parameters $u_{\max }$ and $h_{\mathrm{u}}$. 


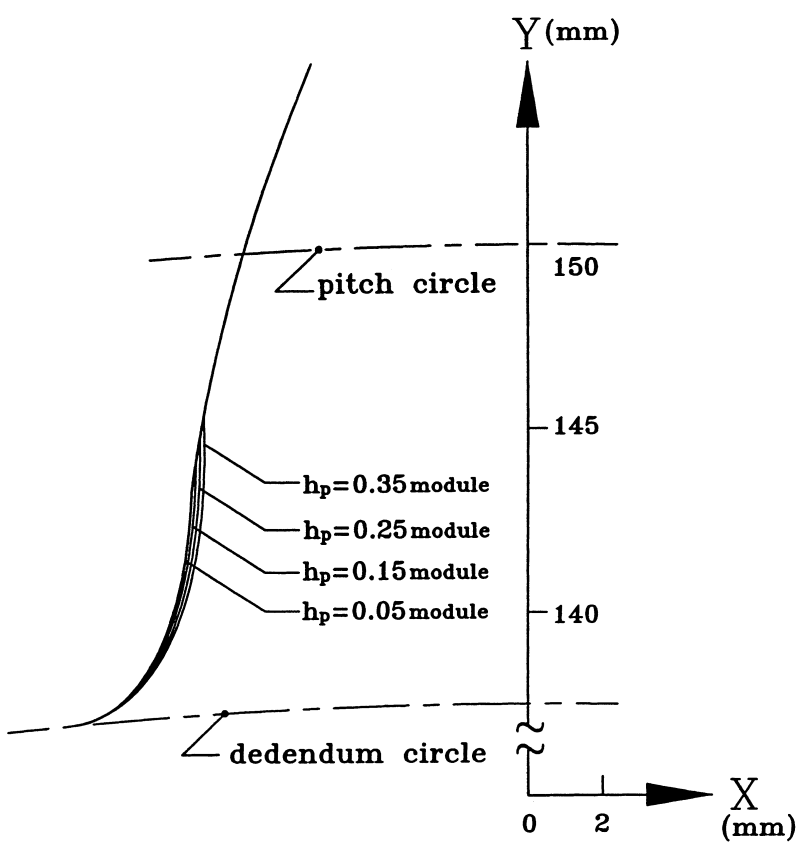

Fig. 8. Effect of protuberance parameter $h_{\mathrm{p}}$ on the generated tooth profile.

According to Fig. 11, $u_{\max }$ and $h_{\mathrm{u}}$ increase with an increase of $\theta_{\mathrm{p}}$. This observation implies that a larger $\theta_{\mathrm{p}}$ induces a larger amount of undercut and reduces the length of the involute working part.

\section{Optimization method for shaper cutter parameters}

In the previous sections, the effect of the shaper cutter parameters $\xi_{\mathrm{d}}, \theta_{\mathrm{s}}, \rho, h_{\mathrm{p}}$ and $\theta_{\mathrm{p}}$ on the generated tooth profile

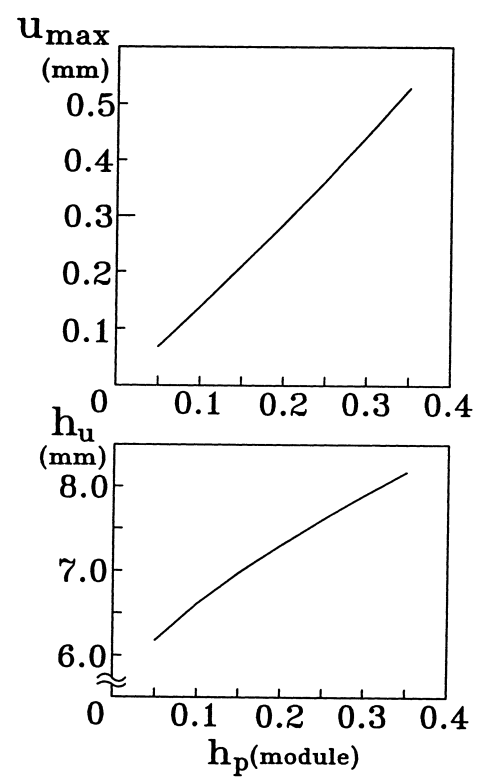

Fig. 9. Effects of protuberance parameter $h_{\mathrm{p}}$ on the fillet parameters $u_{\max }$ and $h_{\mathrm{u}}$.

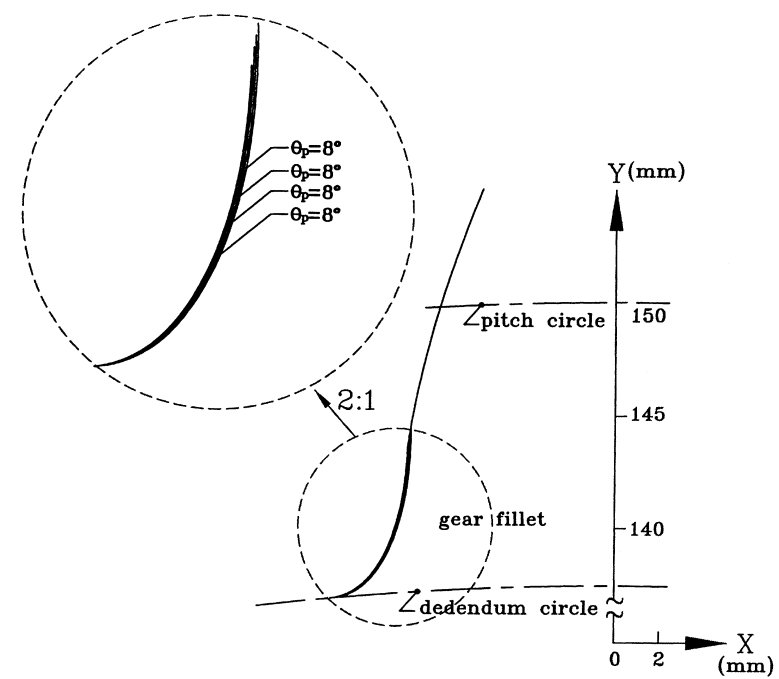

Fig. 10. Effect of protuberance parameter $\theta_{\mathrm{p}}$ on the generated tooth profile.

have been examined individually. However, according to the present results, the relationships among the shaper cutter parameters and the gear parameters are non-linear and complicated. Therefore, an optimization method is employed to determine the proper shaper cutter parameters.

\subsection{Semi-topping parameters}

The semi-topping parameters, $\theta_{\mathrm{s}}$ and $\xi_{\mathrm{d}}$, are defined as the design variables. Under the desired values of chamfer parameters $\theta_{\mathrm{c}}^{*}$ and $h_{\mathrm{c}}^{*}$, the cost function in the optimization problem formulation [12] can be represented by

$\min f\left(\theta_{\mathrm{s}}, \xi_{\mathrm{d}}\right)=\left|\theta_{\mathrm{c}}^{(i)}-\theta_{\mathrm{c}}^{*}\right|+\left|h_{\mathrm{c}}^{(i)}-h_{\mathrm{c}}^{*}\right|$,

where superscript $i$ represents the iteration number during the optimization process. There is no constraint equation in

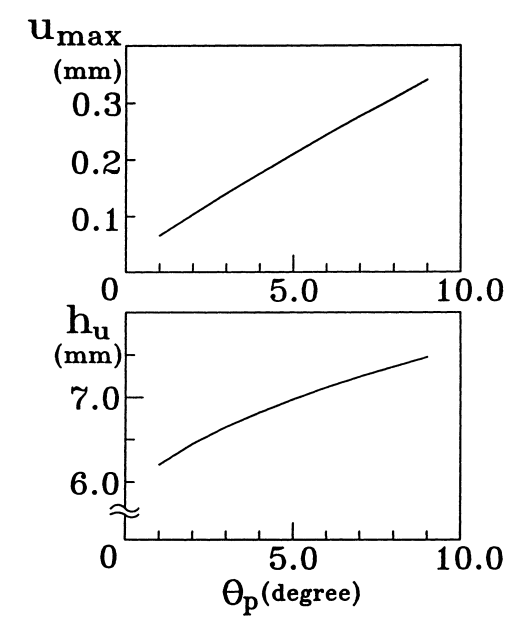

Fig. 11. Effects of protuberance parameter $\theta_{\mathrm{p}}$ on the fillet parameters $u_{\max }$ and $h_{\mathrm{u}}$. 
this optimization formulation. The boundaries of the design variables can be formulated as follows:

$\theta_{\mathrm{s}, \min }<\theta_{\mathrm{s}}<\theta_{\mathrm{s}, \max }$

$\xi_{\mathrm{d}, \min }<\xi_{\mathrm{d}}<\xi_{\mathrm{d}, \max }$.

Reasonable boundaries of the design parameters, $\theta_{\mathrm{s}}$ and $\xi_{\mathrm{d}}$, can be chosen by making reference to the result shown in Figs. 8-11.

\subsection{Protuberance parameters}

The design variables for the protuberance are $\theta_{\mathrm{p}}, h_{\mathrm{p}}$, and $\rho$. Letting the maximum desired amount of the undercut be $u_{\max }^{*}$, the cost function can be represented by

$\min f\left(\theta_{\mathrm{p}}, h_{\mathrm{p}}, \rho\right)=\left|u_{\max }^{(i)}-u_{\max }^{*}\right|$,

where superscript $i$ refers to the iteration number during the optimization process. According to Fig. 1, the radius $\rho$ of curve BC cannot be larger than the protuberance height $h_{\mathrm{p}}$. Fig. 7 reveals that the length of working part decreases with an increase of $h_{\mathrm{u}}$. Therefore, $h_{\mathrm{u}}$ should not exceed the maximum allowable value $h_{\mathrm{u}}^{*}$. Accordingly, the constraint conditions can be written as follows:

$h_{\mathrm{u}} \leq h_{\mathrm{u}}^{*} \quad$ and $\quad \rho \leq h_{\mathrm{p}}$.

The suitable boundaries of the three design variables may be chosen according to the handbook and can be formulated as follows:

$\theta_{\mathrm{p}, \min } \leq \theta_{\mathrm{p}} \leq \theta_{\mathrm{p}, \max }, \quad h_{\mathrm{p}, \min } \leq h_{\mathrm{p}} \leq h_{\mathrm{p}, \max } \quad$ and

$\rho_{\min } \leq \rho \leq \rho_{\max }$

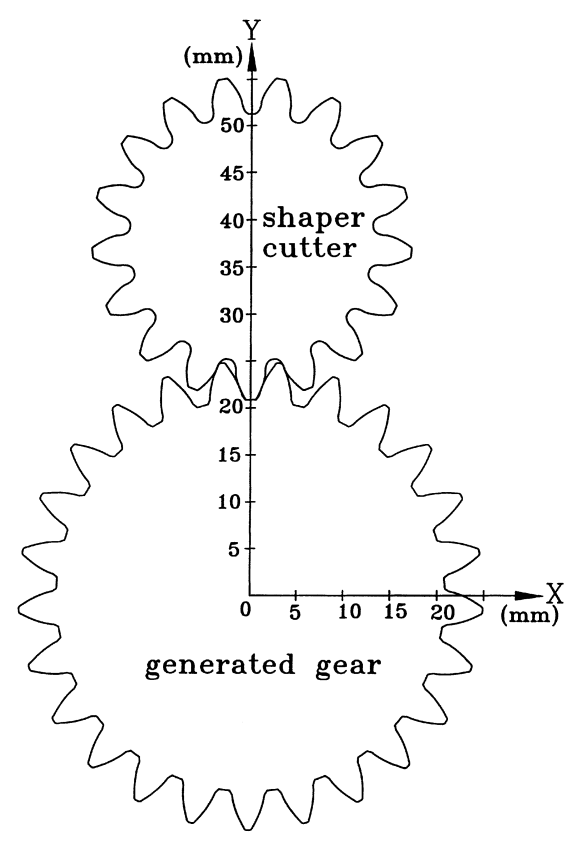

Fig. 12. Computer graphs of the shaper cutter and the generated gear.

\subsection{Numerical example}

Computer programs are developed to facilitate the optimization process based on the developed formulation and the mathematical models of the shaper cutter and the generated gear. Some parameters for the generated spur gear are: teeth number $=17$ teeth, pressure angle $=22.5^{\circ}$, module $=1.814 \mathrm{~mm}$, maximum undercut amount $u_{\max }^{*}=0.04 \mathrm{~mm}$, chamfer height $h_{\mathrm{c}}^{*}=0.1 \mathrm{~mm}$, chamfer angle $\theta_{\mathrm{c}}^{*}=45^{\circ}$ and the value of $h_{\mathrm{u}}^{*}=0.7335 \mathrm{~mm}$.

The teeth number of the shaper cutter is designed to be 40 . Based on the optimization computer program developed, the optimum results of protuberance parameters on the shaper cutter are $\theta_{\mathrm{p}}=4.502^{\circ}, h_{\mathrm{p}}=0.196$ module and $\rho=0.176$ module. On the other hand, the optimum values of semi-topping parameters are $\theta_{\mathrm{s}}=55.26^{\circ}$ and $\xi_{\mathrm{d}}=10.84^{\circ}$. Fig. 12 displays the computergraph of the shaper cutter and the generated spurgear.

\section{Conclusions}

This study presents the complete geometry of a twodimensional shaper cutter for spur gear production, which comprises three major regions: the protuberance (straight line and rounded tip), semi-topping, and the involute curve. Based on the theory of gearing, the mathematical model of the generated spur gear is also derived, including standard and profile-shifted teeth. In addition, several computer graphs illustrate the effects of the protuberance and semitopping parameters on the generated tooth profile. Numerical examples demonstrate that the chamfer height $h_{\mathrm{c}}$ increases with an increase of semi-topping parameter $\xi_{\mathrm{d}}$ (i.e. the involute polar angle of the starting point of semitopping). Further, the chamfer angle $\theta_{\mathrm{c}}$ increases with an increase of semi-topping parameter $\theta_{\mathrm{s}}$ (i.e. the angle formed by the semi-topping and a horizontal line). For the generated fillet, a larger protuberance height $h_{\mathrm{p}}$ results in a more severe undercut. In addition, the undercut becomes more serious with an increase of the protuberance angle $\theta_{\mathrm{p}}$. The amount of intentional undercut also increases with a decrease of the radius of the rounded tip on the protuberance, $\rho$. The present results also indicate that the working region of the generated gear decreases when the undercut becomes more severe. Finally, a numerical method using an optimization technique is applied to determine adequate shaper cutter parameters for achieving the prescribed tooth parameters are given. The mathematical models and computer programs developed herein can help the gear designer to design and choose proper parameters in the shaping of spur gears.

\section{Acknowledgements}

The authors would like to thank the National Science Council of the ROC for financially supporting this research under Contract No. NSC 88-2212-E-009-006. 


\section{References}

[1] S.M. Vijayakar, B. Sarkar, D.R. Houser, Gear tooth profile determination from arbitrary rack geometry, Gear Technol. (November/December 5 (6) 1988) 18-30.

[2] Y. Ariga, S. Nagata, Research on gear cutter tooth profile for semitopping: a case of a helical gear, in: Proceedings of the International Power Transmission and Gearing Conference, DE-Vol. 43 (1), Book No. H0769A, 1992, pp. 423-429.

[3] J.H. Kuang, W.L. Chen, Determination of tip parameters for the protuberance preshaving cutters, Mech. Mach. Theory 31 (7) (1996) 839-849.

[4] R.N. Green, H.H. Mabie, Determination of pinion cutter offsets required to produce nonstandard spur gears with teeth of equal strength, Mech. Mach. Theory 15 (1980) 491-506.

[5] C.A. Rogers, H.H. Mabie, C.F. Reinholtz, Design of spur gears generated with pinion cutters, Mech. Mach. Theory 25 (6) (1990) 623-634.
[6] H. Yoshino, M. Shao, A. Ishibashi, Design and manufacture of pinion cutters for finishing gears with an arbitrary profile, Int. J. JSME Ser. III 35 (2) (1992) 313-319.

[7] J.D. Kim, D.S. Kim, Development of software for the design of a pinion cutter, J. Mater. Process. Technol. 68 (1997) 76-82.

[8] S.L. Chang, C.B. Tsay, Computerized tooth profile generation and undercut analysis of noncircular gears manufactured with shaper cutters, ASME J. Mech. Design 120 (1998) 92-99.

[9] W.L. Janninck, Shaper cutters - design and application, Part 1, Gear Technol. 7 (2) (March/April 1990) 35-48.

[10] W.L. Janninck, Shaper cutters - design and application, Part 2, Gear Technol. 7 (3) (May/June 1990) 38-48.

[11] F.L. Litvin, Gear Geometry and Applied Theory, Prentice-Hall, Englewood Cliffs, NJ, 1994.

[12] J.S. Arora, Introduction to Optimum Design, McGraw-Hill, New York, 1989. 